\title{
Complex extended line emission in the cD galaxy in Abell 2390
}

\author{
J.B. Hutchings ${ }^{1}$ \\ Dominion Astrophysical Observatory \\ Herzberg Institute of Astrophysics, National Research Council of Canada \\ 5071 W. Saanich Rd., Victoria, B.C. V8X 4M6, Canada \\ M.L. Balogh 2,3 \\ Dept of Physics and Astronomy, University of Victoria \\ P.O. Box 3055, Victoria, B.C. V8W 3P6, Canada
}

\begin{abstract}
This paper reports maps of the $\mathrm{cD}$ galaxy in the rich $\mathrm{z}=0.23$ cluster Abell 2390 at UV, [OII], L $\alpha$ and $\mathrm{H} \alpha$ wavelengths. Spatially resolved UV and optical spectra were obtained with STIS on the Hubble Space Telescope; the $2^{\prime \prime}$ wide slit was aligned close to the long axis and blue lane of the galaxy and includes all the inner bright features seen in WFPC2 images. The $\mathrm{L} \alpha$ is seen in emission from several bright knots and over an extended region from $4^{\prime \prime} \mathrm{NW}$ to $2^{\prime \prime}$ SE of the nucleus. Three of these knots have detected UV continuum as well. $\mathrm{H} \alpha$ images were obtained with OSIS at CFHT; both $\mathrm{H} \alpha$ and [O II] show extended emission that roughly trace L $\alpha$, primarily to the NW. Notable differences between the spatial distributions of $\mathrm{H} \alpha, \mathrm{L} \alpha$ and [O II] emission and the blue knots in the galaxy may be the result of inhomogeneous dust extinction, or variations in ionisation. The $\mathrm{L} \alpha$ emission velocities depend the uncertain identification with features in the undispersed images; there is strong evidence for resolved emission knots to have large velocities, $z 1000 \mathrm{~km} / \mathrm{s}$, indicative of infall. The $\mathrm{L} \alpha$ spectrum shows a sharp cutoff which may be due to absorption; we entertain the possibility that this edge is due to an shell of absorbing gas, outflowing at $\sim 5000 \mathrm{~km} / \mathrm{s}$.
\end{abstract}

\footnotetext{
${ }^{1}$ Based on observations with the NASA/ESA Hubble Space Telescope, obtained at the Space Telescope Science Institute, which is operated by AURA Inc under NASA contract NAS5-26555

${ }^{2}$ Visiting Astronomer, Canada-France-Hawaii Telescope, which is operated by the National Research Council of Canada, le Centre Nationale de la Recherche Scientifique, and the University of Hawaii.

${ }^{3}$ Present address: Department of Physics, University of Durham, South Road, Durham, England DH1 $3 \mathrm{LE}$
} 


\section{Introduction}

The central galaxies of rich clusters often differ remarkably from other cluster ellipticals in their morphological and spectroscopic properties; in particular, a large fraction $(\sim 40 \%)$ show strong nebular emission lines and an excess ultraviolet/blue continuum (e.g., Johnstone, Fabian \& Nulsen 1987; Heckman et al. 1989; McNamara \& O'Connell 1993; Crawford et al. 1999). These features are more common in clusters selected from $\mathrm{X}$-ray samples, and especially in those which are known to have strong cooling flows. The optical line emission is generally very concentrated in a central region of only $5-10 \mathrm{kpc}$ (e.g. Heckman et al. 1989; Crawford et al. 1999), sometimes with filaments extending beyond the stellar continuum, $\sim 20 \mathrm{kpc}$ into the intracluster medium (e.g. Cowie et al. 1983; Romanishin \& Hintzen 1988; Crawford \& Fabian 1992). The cooling flow gas itself extends to much larger scales $(\sim 100 \mathrm{kpc})$.

The origin of the nebular emission in cooling flow clusters is uncertain, and is likely not the same in all clusters. Generally, the line luminosity is too strong to arise directly from the cooling gas. Heckman et al. (1989) showed that the spatial variation of emission line ratios generally corresponds better with shock models, rather than photoionization models. Other authors (e.g. Filippenko \& Terlevich 1992, Allen 1995) claim the line ratios can be produced by very massive O-stars, which may form in the collision of cold gas clouds within the cooling flow. The star formation rates determined from this nebular emission, assuming the gas is photoionised by massive stars, are generally $10-100 M_{\odot} \mathrm{yr}^{-1}$ (e.g. Johnstone et al. 1987; McNamara \& O'Connell 1993; Allen 1995; Crawford et al. 1999), which are at least a factor of 10 less than the mass inflows implied by the cooling flows.

The luminous emission line nebulae in these unusual galaxies are often asymmetrically distributed about the galactic nucleus (Crawford \& Fabian 1992). McNamara \& O'Connell (1993) observe strong colour structure in the blue spectra of two such galaxies which have smooth I-band isophotes. In some cases, this structure is seen to correlate with features at radio wavelengths (e.g. Heckman et al. 1989; Edge et al. 1999) which suggest ionisation of the gas is at least partly due to a central radio source. The velocity structure of the nebulae also tends to be complex. Heckman et al. (1989) found that the velocities of $\mathrm{H} \alpha$ nebulae in nine central galaxies are very disordered (uncorrelated with position), with velocities of $\lesssim 200 \mathrm{~km} / \mathrm{s}$. They claim this is consistent with a scenario in which the gas is clumpy and infalling (in a cooling flow), such that the "random" fluctuations in velocity are due to clumps observed in both the foreground and background of the centre. Occasionally much higher velocities are observed; Johnstone \& Fabian (1995) observe Ly $\alpha$ emission and absorption velocities at $1000 \mathrm{~km} / \mathrm{s}$ and greater, which are likely also due to nebulae infalling along the line of sight (Haschick, Crane \& van der Hulst 1982). 
Recent observations have detected large amounts of dust in some central galaxies, often amounting to $E_{B-V} \gtrsim 0.5$ (e.g. Hu 1992; Allen et al. 1995; McNamara et al. 1996; Pinkney et al. 1996; Crawford et al. 1999). The presence of such large amounts of dust may itself require a high star formation rate to replenish the supply destroyed by sputtering (e.g. Draine \& Salpeter 1979). Neglecting this dust when analysing emission line fluxes can lead to an underestimation of the implied star formation rates by an order of magnitude.

The cluster Abell 2390 is host to a particularly strong cooling flow, with an inflow rate of $\sim 800 M_{\odot} \mathrm{yr}^{-1}$ derived from ROSAT observations (Pierre et al. 1996). The galaxy populations in this cluster have been studied in some detail by the Canadian Network for Observational Cosmology (CNOC1) consortium, using images and spectra from the Canada France Hawaii Telescope (Yee et al 1996, Abraham et al 1996); evidence for infall and strong population gradients throughout the cluster is seen. The central bright cD galaxy of the cluster has considerable size and structure and was studied using the CNOC1 database by Davidge and Grinder (1995). It has a lumpy morphology at resolutions of a few arcseconds or better, extremely blue $(g-r)$ and $(U-B)$ colour (Smail et al. 1998) and a predominantly young stellar population, with strong [O II] emission (rest frame equivalent width of $110 \pm 2 \AA$ ). Davidge and Grinder note that, in this respect, this galaxy is similar to the $\mathrm{cD}$ galaxies of CNOC1 clusters $0839+29$ and $1455+22$, and different from other cDs in that survey. This galaxy has also been studied in detail at submillimetre, radio, infrared and optical wavelengths by Edge et al. (1999); in particular, these authors note the presence of a strong blue lane (or "cone") in the archival WFPC2 observations which is oriented orthogonally to the $4.89 \mathrm{GHz}$ radio map, suggesting that the gas in this cone is ionised by a strong nuclear source. Edge et al. and Lemonon et al. (1998) claim that there is some evidence for dust in this galaxy, though this is strongly dependent on the uncertain nature of the ionisation source.

We have obtained HST STIS observations of the cD galaxy in A2390 with a 2" slit, which provides spatial and velocity information about the Ly $\alpha$ and [OII] emission lines, and the UV continuum. We compare these data with CFHT H $\alpha$ observations obtained as part of a larger study (Balogh \& Morris 1999). With this data, we map the structure of nebular emission within $\sim 10 \mathrm{kpc}$ of the galaxy centre. We present our observations in $\S 2$, and our results on the spatial and velocity structure of the emission line gas in $\$ 3$. In $\$ 4$ we discuss some of the implications of these observations. Our results are summarized in $\S 5$.

\section{Observations}




\subsection{STIS Observations}

HST STIS observations were obtained with the $2^{\prime \prime}$ wide slit aligned at $\sim 25^{\circ}$ to the direction of the blue lane seen in the WFPC2 images (\$2.3). Standard reduction procedures (flats and wavelength calibration) were followed. The slit direction was chosen to include another central cluster galaxy, and two of the gravitational arcs near the cluster centre, for additional information. Spectra were taken at both optical and UV wavelengths, as summarized in Table 1. The blue G430L spectrum has resolved [O II] and $\mathrm{H} \gamma$ emission, while the G750L exposure is too short to be useful, though it does show $\mathrm{H} \alpha$ emission and continuum. L $\alpha$ emission is clearly resolved in the FUV MAMA spectra. The very wide slit ensured that all bright central features in the galaxy were included, and the resulting spectra are essentially slitless, so that spectral feature positions depend on their physical location as well as their radial velocities. The STIS observation first centred on a nearby bright star, and then moved to the galaxy by accurate blind offset. Since the galaxy is extended, its coordinates may not be exactly those of the nucleus, and there is some uncertainty (perhaps $\sim 0.1 "$ ) in the exact location of the 2 " wide slit.

\section{2. $\mathrm{H} \alpha$ Imaging}

Images of the $\mathrm{cD}$ galaxy in $\mathrm{H} \alpha+\mathrm{N}[\mathrm{II}]^{\mathrm{f}}$ light were taken with $O S I S$ on the CFHT, with a specially designed interference filter (Balogh 1999, Balogh \& Morris 1999). A continuum image, formed by combining narrow band images redward and blueward of $\mathrm{H} \alpha$, was subtracted from the on-line image, after matching the PSF (0'.8), to produce the final image. Full details of the reduction procedure are given in Balogh (1999). The $\mathrm{H} \alpha$ image was aligned with the WFPC2 images by matching the $\mathrm{H} \alpha$ continuum image with the WFPC2 F814W image, and this should be good to about 0'1. H $\alpha$ fluxes could not be accurately determined, since the nights were not photometric, but equivalent widths are reliably measured. The $\mathrm{H} \alpha$ filter used was quite wide, $324 \AA \mathrm{FWHM}$, so $\mathrm{H} \alpha$ emitted at $v \lesssim 6000 \mathrm{~km} \mathrm{~s}^{-1}$ relative to the $\mathrm{cD}$ galaxy will be detected.

\subsection{Comparison with Archival WFPC2 Images}

We have obtained images of the cD galaxy in Abell 2390 from the HST archive; it has been observed with the WFPC2 with the F555W and F814W filters. Figure 11 shows all of

\footnotetext{
${ }^{4}$ Hereafter, we refer to this as $\mathrm{H} \alpha$ alone; corrections for $\mathrm{N}[\mathrm{II}]$ are made when necessary.
} 
the images with the same spatial scale, and also the width of the slit, the length of which is vertical in the orientation of the diagram. The first panel shows the WFPC2 F555W image, and the third panel presents the F814W/F555W ratio, thus showing the colour distribution (light shades correspond to blue colours). The horizontal, solid line shown in the F555W image represents the size and approximate location of the $2^{\prime \prime}$ slit used to obtain the STIS spectra. At the cluster redshift of $z=0.23,1^{\prime \prime}$ corresponds to $3.4 \mathrm{kp}$. We consider the redder knot that lies in the centre of the general galaxy light to be the nucleus; this feature is surrounded by red material. There is also a red, resolved knot, 23" E of S from the galaxy that is a separate small galaxy of red colour (number 956 in Yee et al 1996). It is a normal-looking elliptical with an old stellar population, and the spectra and $\mathrm{H} \alpha$ images do not reveal any spatially varying properties or line emission. It is too far away from the $\mathrm{cD}$ galaxy to appear in the UV spectral image, which covers only $25^{\prime \prime}$. There is a faint signal at the position of the bright arc about $8^{\prime \prime} \mathrm{S}$ of the $\mathrm{cD}$, which is flat and without strong emission in the observed blue wavelength range. This is consistent with a very blue stellar population, at redshift larger than 0.23 . There is no significant signal at the position of a larger red arc about $14^{\prime \prime}$ from the $\mathrm{cD}$ galaxy. We do not discuss these other objects further.

\section{Results}

\subsection{Spatial Structure}

The most prominent blue features seen in the WFPC2 images of the $\mathrm{cD}$ galaxy in Figure 1 are the resolved knots below the nucleus, just above the nucleus, and the linear feature above the nucleus. These all lie roughly along a line. Edge et al (1999) interpret the blue lane as a double "conical" feature, similar to that seen in Seyfert galaxies, and suggest that this material may be ionised by the strong nuclear source.

The UV spectrum shown in the right hand panel of Figure 1 (and, spatially compressed, in Figure 2 1) shows a remarkable L $\alpha$ complex. The direct and dispersed images are lined up exactly in the spatial direction along the slit. The $2^{\prime \prime}$ slit covers the brightest, central region ( $\sim 7 \mathrm{kpc}$ ) of the galaxy and its various bright knots; the continuum profile of the halo itself has an effective radius of about $10^{\prime \prime}$. There is $\mathrm{L} \alpha$ emission that extends over the whole length of the inner galaxy, and is considerably more extended below (NW) the nucleus, beyond the bright blue knots. The brightest $\mathrm{L} \alpha$ knots are the nucleus itself and the knots above and below it in our image.

\footnotetext{
${ }^{5}$ We assume a cosmology with $\mathrm{H}_{\circ}=75 \mathrm{~km} \mathrm{~s}^{-1} \mathrm{Mpc}^{-1}, \Omega_{\circ}=0.3$ and $\Omega_{\Lambda}=0.7$.
} 
There is UV continuum from three of the knots, including one fainter blue knot below the nucleus. However, continuum light from the nucleus itself is not detected. The CCD blue spectral image shows that there is continuum at the nucleus and the bright knots above it in our diagram, but not from the knot below which is bright in the UV. The [O II] line emission is brightest at the nucleus but is seen clearly at the two L $\alpha$ knots as well. The blue lane does not correspond with either line or continuum flux in our data, so presumably arises from a combination of lines and continuum in the WFPC2 passbands.

There is a prominent, short wavelength edge to the L $\alpha$ emission. This is shown more clearly in Figure 2, which is compressed along the spatial direction to make this edge and its curvature more apparent. Where the edge crosses the lower continuum region, there is some evidence for absorption over an interval shortward of the edge; this can be seen in spectral profile shown in Figure 3. However, the shape of the continuum is not well defined, which makes this determination uncertain.

As noted above, in contrast to the $\mathrm{L} \alpha$ image, the nucleus is the brightest feature in the dispersed [O II] image. Furthermore, no evidence for the sharp L $\alpha$ edge is seen in the [O II] profile, as shown in Figure 3. We note that the [O II] profile we show is predominantly that of the nuclear region: the contribution from the $\mathrm{L} \alpha$ bright knot is small and does not affect the shortward side of the profile we show. The presence of [O II] emission at velocities more negative than those at $\mathrm{L} \alpha$ suggests that the $\mathrm{L} \alpha$ edge may be due to absorption, since the [O II] doublet (velocity separation $\sim 220 \mathrm{~km} \mathrm{~s}^{-1}$ ) is from forbidden transitions. However, the [OII] emission signal is too weak to enable us to draw strong conclusions. The velocity structure of the $\mathrm{L} \alpha$ edge and of the various emission features is discussed in more detail in $\S 3.2$.

The image of the $\mathrm{cD}$ galaxy in $\mathrm{H} \alpha$ light is shown in the left hand panel of Figure $\mathbb{1}$. The nuclear regions of the galaxy, and at least two regions extending several arcseconds below (NW) the nucleus, are clearly detected. The photometric zero point of the H $\alpha$ image is uncertain by at least $\sim 0.3 \mathrm{mag}$, as observing conditions were poor; the rest frame equivalent width line (which is independent of this zero point) is $150 \pm 7 \AA$ within a $5^{\prime \prime}$ diameter aperture.

The nuclear region is extended towards the knot above (in Figure 1) the nucleus, but does not show a peak at the same position. The first $\mathrm{H} \alpha$ emission region below the nucleus corresponds with the faint inner knots in the WFPC2 image, and extends towards the brighter blue knot below. The absence of detected $\mathrm{H} \alpha$ emission to the $\mathrm{SE}$ of the nucleus, along the clearly visible blue lane, is notable. However, the $\mathrm{H} \alpha$ data are not very sensitive; the limiting $\mathrm{H} \alpha$ magnitude is $m_{A B}=21.7$ (for a $2 \sigma$ point source detection), which corresponds to a flux limit of $1.6 \times 10^{41} \mathrm{ergs} / \mathrm{s}$. Weaker emission may be present along the 
blue lane; clearly, however, the strongest emission originates from the NW of the nucleus.

The L $\alpha$ bright knot emission distribution appears to correspond more closely with the features in the HST continuum images, rather than the $\mathrm{H} \alpha$ image. However, this comparison is complicated by the very different spatial resolutions, and the faintest emission boundaries do resemble those of $\mathrm{H} \alpha$ if we assume that the slit edge cuts off the leftmost part of the L $\alpha$ emission. The $\mathrm{H} \alpha$ emission peak farther to the NW extends towards the two fainter blue knots farthest from the nucleus, but does not correspond in detail with their positions. Plots of the row-averaged light from the $\mathrm{H} \alpha, \mathrm{F} 555 \mathrm{~W}$, and $\mathrm{L} \alpha$ images (not shown) confirm that they differ significantly. In particular, the L $\alpha$ emission is strong at the blue knots with UV continuum, while $\mathrm{H} \alpha$ is not; there are other places where the reverse is true. Similar effects are seen in nearby starburst galaxies, which Conselice et al. (1999) claim are consistent with a picket fence dust distribution. However, it is still unclear whether, in this case, the observed structure is mostly due to differences in extinction, ionisation, or sensitivity.

We note finally that we have a VLA C-configuration $1.4 \mathrm{GHz}$ map of the galaxy that shows a weak extension in the general direction of the NW knots. Edge et al (1999) claim that the $\mathrm{A}$ and $\mathrm{B}$ configuration reveal a smaller $\left(0.3^{\prime \prime}\right)$ extension at $5 \mathrm{GHz}$, possibly normal to the blue lane. Deeper, high resolution radio maps at several frequencies would be of interest in understanding whether there are jets along the blue lanes or not.

\subsection{Velocity Structure}

Because of the wide slit, there is ambiguity between spatial and velocity structure in the STIS spectra which plagues our analysis of the nebular velocities. The geocoronal emission produces an emission line that is $50 \AA$ wide, from uniformly distributed emission at zero radial velocity. A similarly distributed $\mathrm{L} \alpha$ emission wider than the $2^{\prime \prime}$ wide slit around the galaxy would produce the same uniformly illuminated spectral feature from

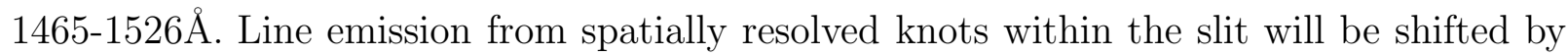
the combination of radial velocity and position within the slit, broadened by the intrinsic motions and spectroscopic resolution.

To measure the velocities of resolved knots, which one can hope to locate spatially from continuum images, we matched the dispersed and undispersed image features in the dispersion direction and noted the relative offsets for as many recognisable components as

possible. This removes the spatial shifts from the dispersed image before measuring velocity shifts, a technique that has been used and described e.g. in Hutchings et al (1998) for 
NGC 4151 slitless data. The quantitative measures were obtained by superposing contour plots of knots in the dispersed and undispersed images. This does not provide an absolute scale, so the zero point was set by identifying the nucleus as described above and referring all other velocity shifts to it. The nuclear velocity is assumed to have the ground-based redshift 0.2301 (Abraham et al 1996) derived from a narrow slit spectrum of the central galaxy. (Yee et al 1996) published a redshift of 0.23024. For the purpose of this paper, the difference, which amounts to some $40 \mathrm{~km} \mathrm{~s}^{-1}$ is not significant, and is within the quoted errors of Yee et al.)

This process assumes that $\mathrm{L} \alpha$ emission, aside from the brightest knots, arises at the peak of the F555W image flux - in particular, along the blue lanes on either side of the nucleus. We regard this as reasonable given the correspondence in overall flux distributions along the galaxy. However, this can only be confirmed by narrower slit observations. If, for example, the $\mathrm{L} \alpha$ emission arises along the faint extensions in the $\mathrm{H} \alpha$ image to the upper left and lower right, rather than the continuum flux along the blue lanes, then the emission velocities will be quite different. Figure $\$$ shows the emission knot velocities based on the blue continuum image and also the $\mathrm{H} \alpha$ image. The values derived from the blue continuum image form a rough S-shape, symmetrical about a point 0 '.2 below the nucleus, about mid-way between the outer UV-continuum knots. The central region has a 'rotation' curve of amplitude about $400 \mathrm{~km} \mathrm{~s}^{-1}$. Further out, this curve reverses, reaching a full amplitude more than $7000 \mathrm{~km} \mathrm{~s}^{-1}$ across the outer regions. Weaker evidence for such high velocities are also present in the [OII] spectra. The velocities we derive if the L $\alpha$ arises in the $\mathrm{H} \alpha$ regions (dots in Figure 4) are also high. Note that the dots in Figure 4 at positions $<-1^{\prime \prime}$ indicate double-valued velocities, since the $\mathrm{H} \alpha$ emission splits and forms two "tails" in this region. This significantly reduces the velocities in the SW, where identification of L $\alpha$ with the blue lane is most in doubt. However, the measured velocities corresponding to the knots are still quite high, and probably arise in in- or out-flowing material.

The curved $L \alpha$ edge is a particularly striking feature. It corresponds closely to the position of the edge of the slit, and this could give rise to this feature if there is extended $\operatorname{L} \alpha$ emission across much of the galaxy, with weak velocity structure to produce the curvature. It is difficult to know the slit position exactly as the geocoronal line is very strong and has broad wings. In addition, there is a zero point shift for the whole velocity scale, derived from a different narrow slit, and there is some uncertainty in the precise redshift from an extended source like this galaxy. However, if the L $\alpha$ edge is caused by the slit position, the curvature and other morphology seen in the dispersed image likely reflects primarily the spatial structure of the ionised gas; the overall shape of the faintest emission is similar for undispersed $\mathrm{H} \alpha$ and dispersed $\mathrm{L} \alpha$, suggesting that this may be the case. 
However, there are some reasons to believe that the sharp edge corresponds instead to a real absorption feature:

1. The line emission is not evenly distributed, but generally peaks near the shortward edge and falls off slowly towards longer wavelengths. The emission image has no redward edge although we might expect emission to cover that part of the galaxy, especially at the top of our diagrams, where the galaxy tilts to that side. If this is spatial structure, we have the unlikely situation where emission arises preferentially toward the slit.

2. The shortward edge is not straight, but has smooth curvature over a large distance. Since it appears to curve in a "C" shape, rather than the "S" shape characteristic of rotation curves, it is difficult to reconcile this with velocity structure.

3. There are dips in the continuum sources shortward of the emission edge, particularly the lowest one in the diagram, indicative of absorption. However, the signal is weak and it is difficult to interpolate the continuum in the plots, as shown in Figure 3; therefore we can only claim that there is a suggestion of real absorption, with low significance.

4. The [O II] image does not show a shortward edge, as expected if the L $\alpha$ edge is due to absorption, since [O II] is a forbidden line. While the signal is low, the emission does have wings that lie in the velocity region "shortward" of the edge in L $\alpha$. While quite suggestive, we note that the absence of an edge in [OII] could also be explained if the [OII] emitting gas is distributed differently (in velocity or position) from that of L $\alpha$.

Given this tentative evidence, we suggest as an alternative that the edge is a true absorption edge, due to an expanding envelope that lies across the whole line of sight. A similar phenomenon is seen in L $\alpha$ observations of $\eta$ Carinae: a curved shifted absorption edge over the length of the extended emission region (Gull, private communication). While we do not suggest strong similarities, it may be that the $\mathrm{cD}$ galaxy is surrounded by an outward-moving wind with a terminal velocity that is well-defined and about the same over all directions covered by the line of sight. The curvature could arise by projection effects if the expansion were at a constant velocity and in a spherical envelope.

To establish the shortward edge velocity under this assumption, we use the wavelength scale from the standard wavelength calibration for the STIS spectrum, with a zero point offset for the wide slit obtained by setting the centre of the strong and sharply-edged geocoronal $\mathrm{L} \alpha$ emission at $1215.7 \AA$. The absorption edge wavelength was measured from plots of sections in the spectral image, as the $10 \%$ point up the short wavelength side of the emission (see examples in Figure 3). The absorption edge velocities were mapped as far as emission is detected in both directions away from the nucleus. 
These edge measurements are shown in Figure 4, referred to as velocities with respect to the overall galaxy redshifted wavelength. This implies an expansion velocity of about $5000 \mathrm{~km} \mathrm{~s}^{-1}$. If we fit a simple expanding spherical absorber model to the curve, moving outwards at this velocity, its radius is about $12 \mathrm{Kpc}$. This is not very sensitive to the zero point of the expansion: at $6000 \mathrm{~km} \mathrm{~s}^{-1}$, the implied radius is $13 \mathrm{Kpc}$. However, if the emission arises spatially along the edge of the slit, we may have velocities as low as 0 $\mathrm{km} \mathrm{s}^{-1}$, with respect to the galaxy mean redshift. The absorption trough seen against the continuum regions appears to extend to some $10000 \mathrm{~km} \mathrm{~s}^{-1}$, but its outer limit cannot be determined very well since the trough becomes weaker as it merges with the background or the weak UV continuum where present (see Figure 3).

\section{Discussion}

There is probably a mixture of distributed emission in this galaxy, including diffuse emission spatially slanted relative to the slit, and resolved emission from the bright knots. We require narrower slit observations to untangle the situation; also, it would be of great interest to obtain undispersed images in $\mathrm{H} \alpha$ and [O II] or [O III] at HST resolution to map the gas in the required detail.

We note that the shortward edge in $\mathrm{L} \alpha$ is not centred on what we regard as the nucleus, but some 0 '.6 below it in our diagrams. The edge wavelength (outflow velocity?) is most extreme next to the brightest UV source, so that possibly this represents the central force driving any outflow. This knot accounts for $20 \%$ of the $\mathrm{L} \alpha$ flux and has magnitude close to 22.3 in both filters (V and I), corresponding to absolute magnitude -18 (similar to the luminosity of the SMC). If this emission is due to photoionisation by massive stars, it is of interest to see if the implied star formation rate can provide enough energy to drive the purported outflow. From the $\mathrm{H} \alpha$ photometry, we use the relation of Kennicutt (1998) to estimate star formation rates (assuming an extinction of $1 \mathrm{mag}$ at $\mathrm{H} \alpha$ and an [NII] $/ \mathrm{H} \alpha$ ratio of 0.5 (Kennicutt 1992)). This results in a star formation rate for the nucleus of $28 \pm 5 M_{\odot} \mathrm{yr}^{-1}$, where the uncertainty reflects the estimated zero point uncertainty. Star formation rates of the same order of magnitude are measured in the two other resolved knots. We calculate the total energy released by supernovae, assuming a frequency of 0.005 per solar mass formed, a total energy per event of $10^{51}$ ergs, and that $10 \%$ of this energy is transfered to the gas. Thus, a star formation rate of $30 M_{\odot} \mathrm{yr}^{-1}$ corresponds to an energy release of $1.5 \times 10^{49} \mathrm{ergs}_{\mathrm{yr}^{-1}}$; this amounts to copious amounts of energy if the burst lasts several 100 Myr. However, this energy must go into not only the kinetic energy of the gas, but to overcoming the $\mathrm{cD}$ gravitational potential and the pressure of the intracluster 
medium (ICM). It turns out that the latter is the most significant. Assuming an isothermal gas density profile, the gas pressure is given by:

$$
P=\frac{1}{2 \pi G}\left(\frac{k T}{\mu m_{H}}\right)^{2} \frac{\Omega_{b}}{\Omega_{\circ}} r^{-2},
$$

where $\mathrm{kT}=9.5 \mathrm{keV}$ is the gas temperature (David et al. 1993), $\mu=0.59$ is the mean molecular weight of the gas, $\Omega_{b}=0.0125 h^{-2}$ is the baryon density parameter predicted by nucleosynthesis (Copi, Schramm \& Turner 1995), the total matter density $\Omega_{\circ}=0.3$, and $r$ is the distance from the centre of the cluster. The total resistant force on an expanding sphere of material $\left(4 \pi r^{2} P\right)$ is therefore independent of distance. The work needed to push a shell of material out to $r=12 \mathrm{kpc}$ against this pressure is $1.0 h^{-2} \times 10^{61} \mathrm{ergs}$; this is much more energy than is available from star formation alone (even if we consider additional energy from stellar winds). If the expanding shell is a real structure, there must be another source of energy driving it, or the ICM pressure must be much lower than we have estimated (as will be the case if, for example, the gas density is much lower than that of an isothermal sphere).

The origin of the blue lane to the SE of the nucleus is uncertain. If the L $\alpha$ detected to the upper left in Figure 1 is associated with this lane (as we assume for the solid line in Figure 4 ), this gas is approaching at very high velocity. However, $\mathrm{H} \alpha$ is only strongly detected to the NW, where the gas velocity is clearly receding. It would be unusual to detect only the receding side of a double jet system in $\mathrm{H} \alpha$. It seems likely, then, that the blue knots and nebular emission arising from the NW are due to infalling nebulae, and that the $\mathrm{L} \alpha$ to the SW is not associated with the blue lane to the SE. It is more likely that the $\mathrm{L} \alpha$ traces the faint $\mathrm{H} \alpha \mathrm{SW}$ extension, and that the linear, blue feature is not associated with any of the emission features. However, narrower slit observations are required to establish this with certainty. One possible explanation is that the blue lane corresponds to a conical hole blown through the intergalactic medium by an active nucleus; if this hole is devoid of dust, and/or lined with hot stars, it may appear blue, but without nebular emission lines.

\section{Summary}

Our data show that there is extended line emission in $\mathrm{L} \alpha, \mathrm{H} \alpha$, and [OII] in the $\mathrm{cD}$ galaxy at the centre of Abell 2390. This emission is seen distributed non-uniformly across and around the galaxy, in both diffuse and knotty components. It is difficult to separate the spatial and velocity shifts in our dispersed images, due to the wide slit; however, there is strong evidence for high velocities due to infalling nebulae. There is a sharp edge in the $\mathrm{L} \alpha$ spectrum which, if due to absorption, may indicate outflow from the whole galaxy with 
very high velocity. It is unlikely that the star formation rates derived from the $\mathrm{H} \alpha$ flux produce enough energy (through supernovae) to overcome the ICM pressure and produce such an outflow.

If the detected line emission arises from massive star formation, this implies that there is ongoing evolution of the cD galaxy. Young populations are found only in a few CNOC1 clusters by Davidge and Grinder (1996), and are rare in low redshift rich clusters (e.g. Oegerle and Hoessel 1991). These data provide additional evidence that clusters with strong cooling flows detected in X-rays have strong emission lines, with asymmetric distributions and spatially varying line ratios (e.g. Crawford et al. 1999). We suggest that the difference between the $\mathrm{H} \alpha$ and $\mathrm{L} \alpha$ images is the result of inhomogeneous dust extinction.

More detailed spectral mapping in other lines with high spatial resolution would be valuable in determining the precise velocity structure of this system. It would also be valuable to extend the sample of such observations to include $\mathrm{cD}$ galaxies with a wide range of line intensity, to quantify the connection between star-formation, inflows/outflows, and cooling flows. In particular, it would be of interest to study the other two CNOC1 cD galaxies with similarities to that of A2390, 0839+29 and 1455+22 (Davidge \& Grinder 1996), in similar detail.

We thank Tim Davidge for helpful discussions on the results in this paper, and Alastair Edge for a careful reading of the manuscript and many useful suggestions. When this work was begun, MLB was supported by a Natural Sciences and Engineering Research Council of Canada (NSERC) research grant to C. J. Pritchet and an NSERC postgraduate scholarship. MLB is currently supported by a PPARC rolling grant for extragalactic astronomy and cosmology at Durham. 


\section{Captions to Figures}

Fig. 1. - Four aligned images of the cD galaxy in A2390. ¿From left to right: (1) H $\alpha$ CFHT image; (2) F555W from WFPC2; (3) ratio F814W/F555W (light=blue); (4) The dispersed image of $\mathrm{L} \alpha$ matched in spatial scale. The solid, horizontal line in the second panel is $2^{\prime \prime}$ long, and shows the width and placement of the slit; the position angle is $154^{\circ}$.

Fig. 2.- The UV spectral image of the cD galaxy of A2390 slightly smoothed and compressed by a factor of 2 in the spatial direction. The wavelength coverage is roughly $1330 \AA$ to $1650 \AA$. Note the three continuum sources and the curved absorption edge to the L $\alpha$ emission structure.

Fig. 3.- Line profiles from STIS spectral images. Note the sharp shortward cutoff seen in L $\alpha$ but not [O II]. The L $\alpha$ knot has a continuum, sketched in roughly, possibly indicating absorption out to velocities of about $-10000 \mathrm{~km} \mathrm{~s}^{-1}$. All velocities refer to the galaxy mean redshift of 0.2301 , and errors are $100 \mathrm{~km} \mathrm{~s}^{-1}$ or less.

Fig. 4.- Velocities of measured L $\alpha$ features, relative to the $\mathrm{cD}$ galaxy. The connected short dashes are emission features spatially identified with the blue continuum peaks. If, instead, the $\mathrm{L} \alpha$ emission traces the $\mathrm{H} \alpha$ emission, the velocities are given by the dots. Vertical lines represent the locations of detected UV continuua. The dashed curve is the inner L $\alpha$ edge velocity, assuming that it is an absorption feature and that the emission arises in the galaxy center or knots.

Table 1. Slit $52 \times 2 \operatorname{arcsec}, \mathrm{PA} 154^{\circ}$

\begin{tabular}{clll}
\hline \hline HST \# & Grating & Exposure & Comment \\
\hline O4VY01070 & G430L & $1500 \mathrm{sec}$ & CCD \\
O4VY01080 & G750L & $150 \mathrm{sec}$ & CCD, v weak \\
O4VY010B0 & G140L & $2300 \mathrm{sec}$ & FUV MAMA \\
O4VY010C0 & G140L & $2300 \mathrm{sec}$ & FUV MAMA \\
\hline
\end{tabular}




\section{References}

Abraham R.G., et al , 1996, ApJ, 471, 694

Allen S. W. 1995, MNRAS, 276, 947

Allen S. W., Fabian A. C., Edge A. C., Bohringer H., White D. A. 1995, MNRAS, 275, 741

Balogh, M. L. 1999, Ph.D. thesis, University of Victoria

Balogh, M. L., Morris, S. L. 1999, in preparation

Conselice, C. J., Gallagher, J. S., Calzetti, D., Homeier, N., Kinney A. 1999, ApJ, accepted (Astro-ph-9910382)

Copi C. J., Schramm D. N., Turner M. S. 1995, Science, 267, 192

Cowie L. L., Hu E. M., Jenkins E. B., York, D. G. 1983, ApJ, 272, 29

Crawford C. S., Allen, S. W., Ebeling, H., Edge, A. C., Fabian, A. C. 1999, MNRAS, 306,857

Crawford C. S., Fabian A. C. 1992, MNRAS, 259, 265

David L. P., Slyz A., Jones C., Forman W., Vrtilek S. D., Arnaud K. A. 1993, ApJ, 412,479

Davidge T.J. and Grinder M., 1995, AJ, 109, 1433

Draine B. T. \& Salpeter E. E. 1979, ApJ, 231, 77

Edge A. C., Ivison, R. J., Smail, I., Blain, A. W., Kneib, J.-P. 1999, MNRAS 306, 599

Filippenko A. V., Terlevich R., 1992, ApJ, 397, L79

Haschick A. D., Crane P. C., van der Hulst, 1982, ApJ, 262, 81

Heckman T. M., Baum S. A., van Breugel W. J. M., McCarthy P., 1989, ApJ, 338, 48

Hu E. M. 1992, ApJ, 391, 608

Hutchings J.B. et al 1998, ApJ, 492, L115

Johnstone R. M., Fabian A. C., Nulsen P. E. J. 1987, MNRAS, 224, 75

Kennicutt, R. C. 1992, ApJ, 388, 310

Kennicutt, R. C. 1998, ARA\&A 36, 189 
Lemonon, L., et al 1998, A\&A, 334, L21

McNamara B.R., O'Connell R. W., 1993, AJ, 105m 417

McNamara B. R., Wise M., Sarazin C. L., Jannuzi B. T., Elston R., 1996, ApJ, 466, 66

Oegerle W.R., and Hoessel J.G., 1991, ApJ, 375, 15

Pierre M., Le Borgne, J.-F., Soucail, G., Kneib, J.-P. 1996, A\&A, 311, 413

Pinkney J., et al. 1996, ApJ, 468, L13

Romanishin W., Hintzen P., 1988, ApJ, 227, 131

Smail I., Edge A. C., Ellis R. S., Blandford R., 1998, MNRAS, 293, 124

Yee H.K.C., et al 1996, ApJS, 102, 289 


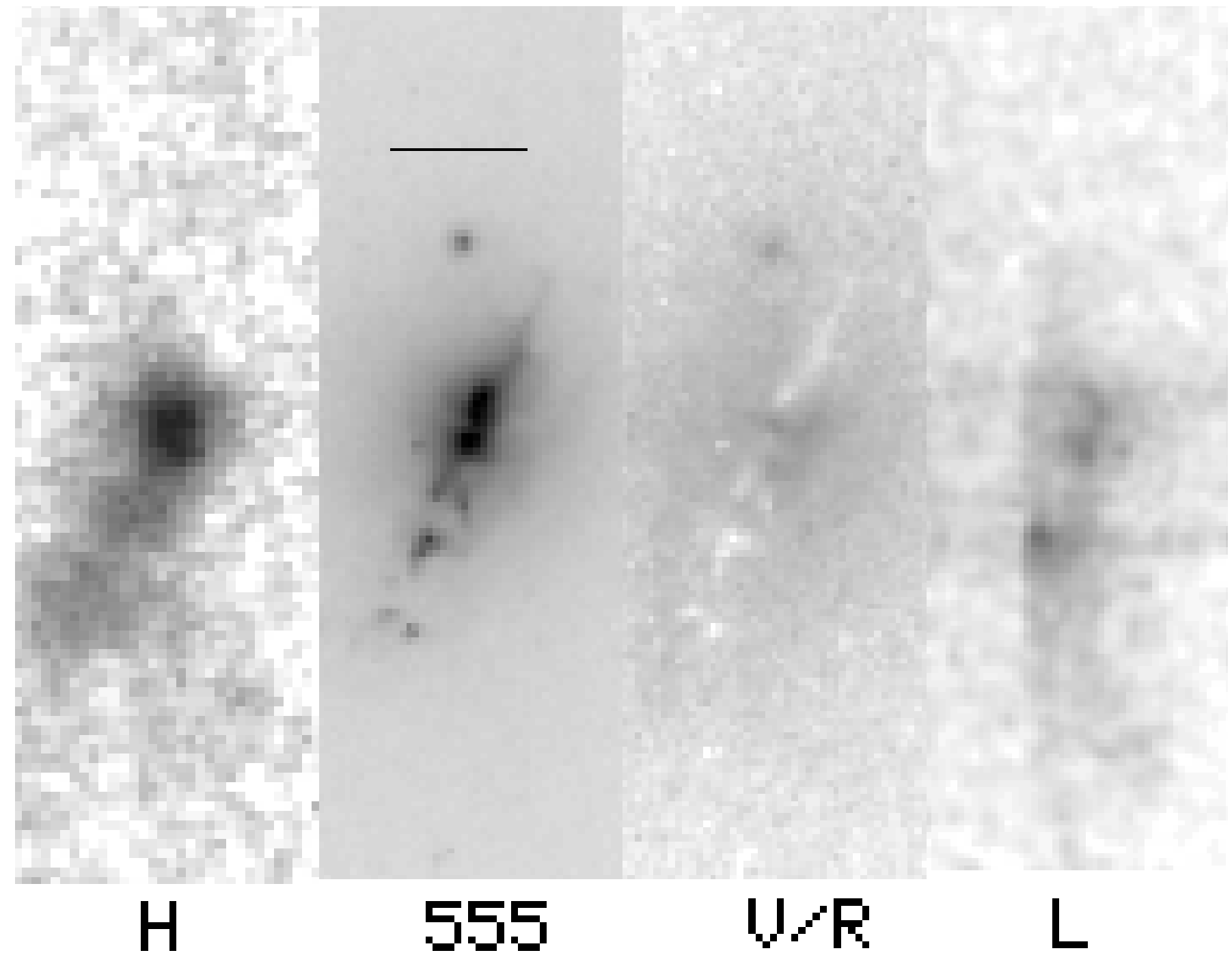




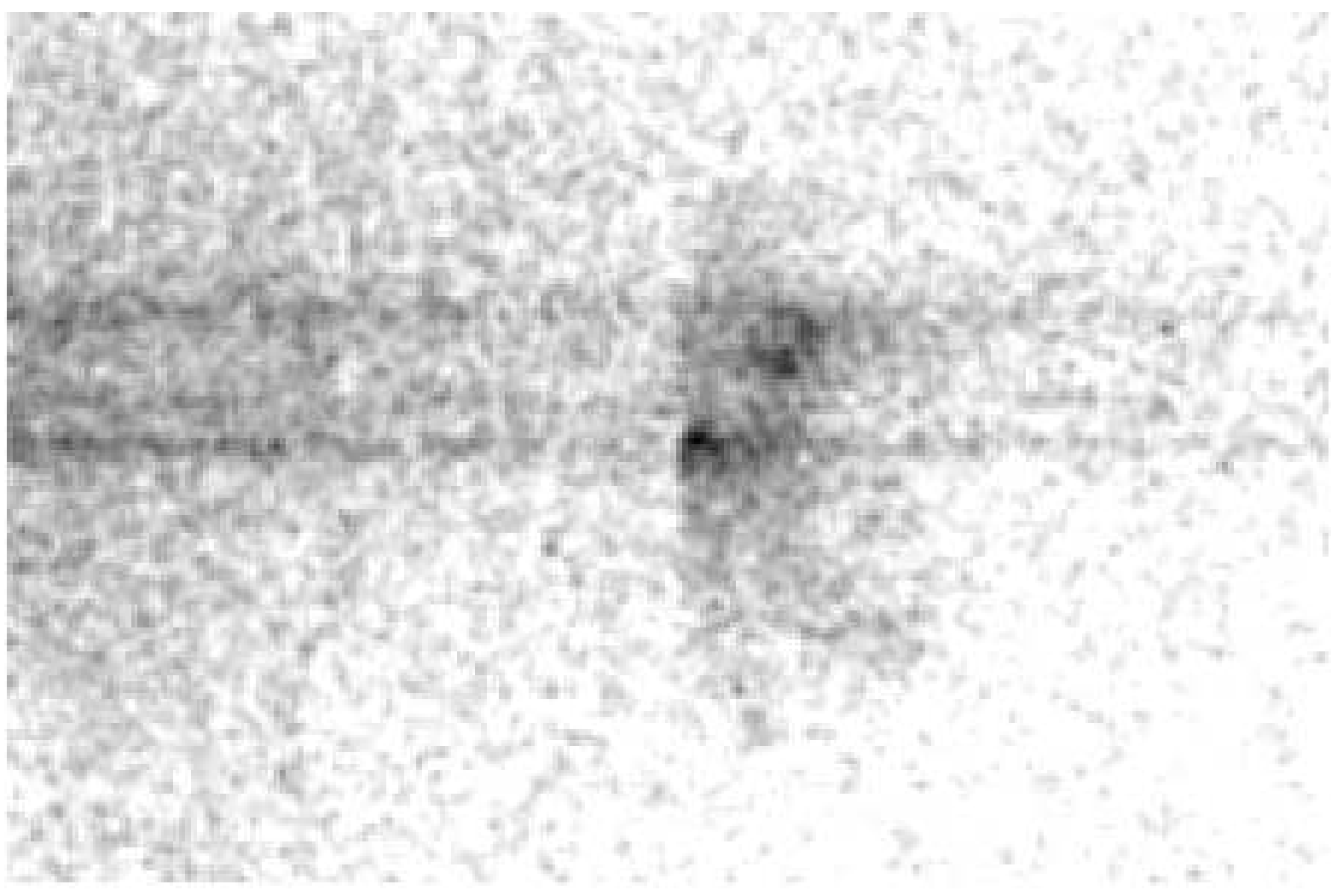


Flux (arb)

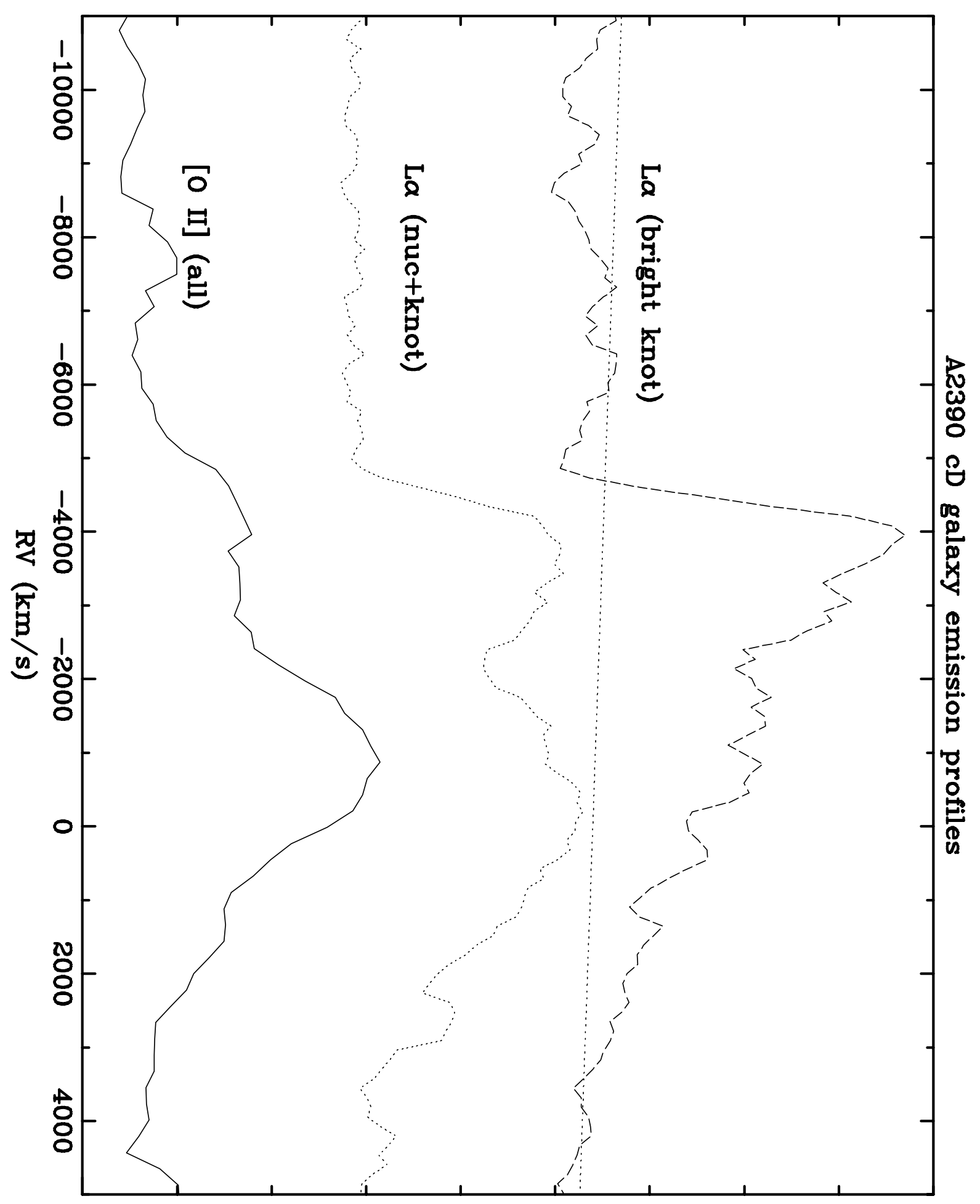




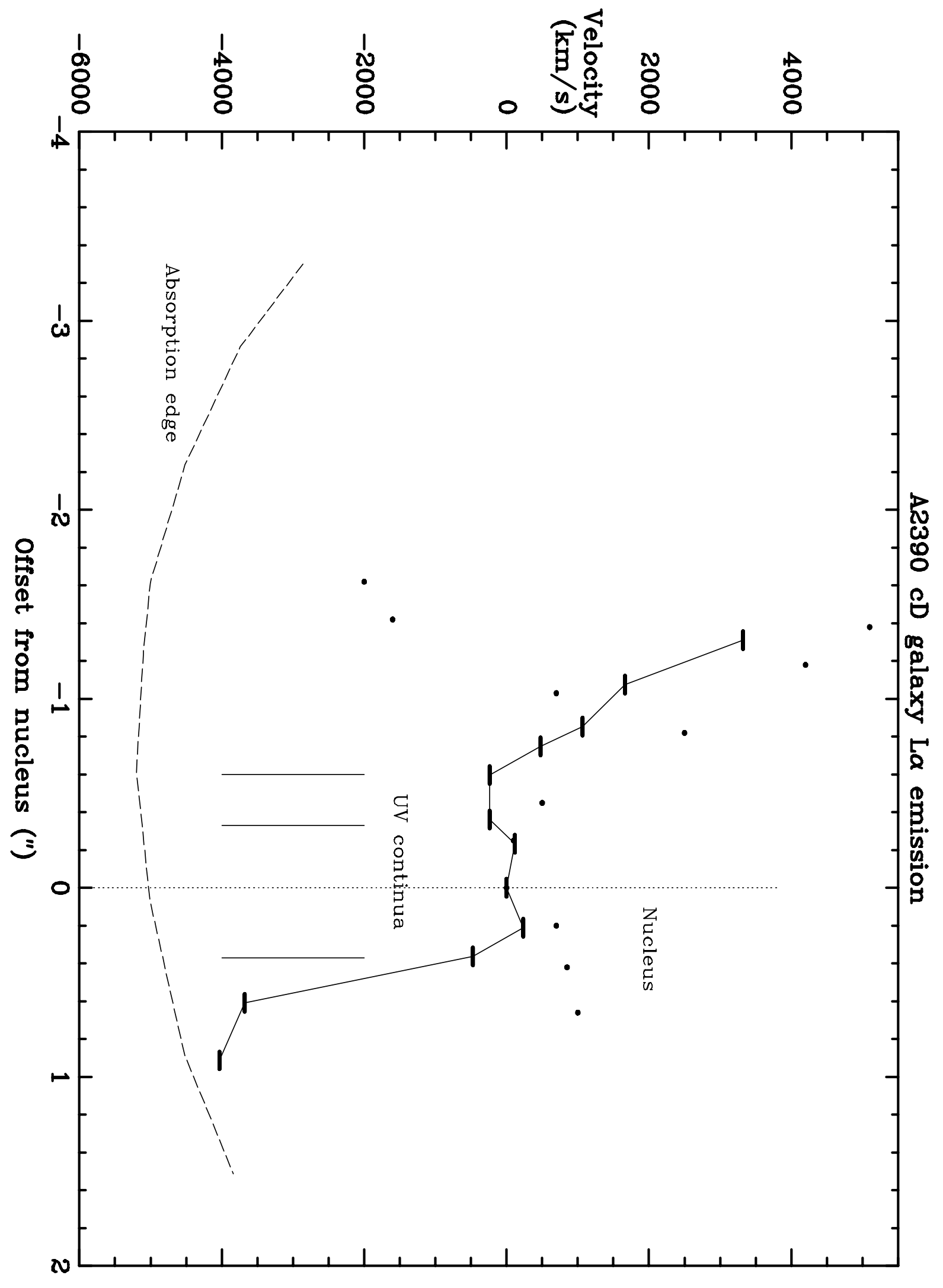

